\title{
Fingerprint Space Building Algorithm with Kriging for Large Positioning Regional Environment
}

\author{
WANG yue, CUI Wei-jia, WANG Da-ming, LU zhi-yu \\ Information Engineering University, Information System Engineering Institute, Zhengzhou,China \\ wangyue302@126.com
}

Keywords: Location Fingerprinting; Kriging; Variogram; Least-Square Fitting

\begin{abstract}
When using the arithmetic of location fingerprinting to build up fingerprint spaces, the methodology that scholars used now contains drawbacks, such as the high complexity and low accuracy and other iuess. As a result, Kriging are using frequently. In the traditional style, chosen Kriging index could only be applied to empirical models for small-scaled indoor scenes. Therefore, proposes to use least-square fitting based variogram model to obtain the theoretical model of related parameters for large targeted area environment. Result of this experiment shows that the new method can improve the fitting degree. Supposing the space of samples is 5-25 meters, the interpolation accuracy enhanced by $0.17 \mathrm{~dB}-0.45 \mathrm{~dB}$, positioning accuracy positioning accuracy are enhanced by $0.4 \mathrm{~m}-1.47 \mathrm{~m}$ as well compared with existing techniques.
\end{abstract}

\section{Introduction}

At present, Location Fingerprinting mainly applies to small indoor positioning scenario. But with the advent of new algorithms and new technologies, domestic and foreign scholars began to utilize Location Fingerprinting to the wider location fields, so that there are two main research directions appeared : extending positioning range and improving the positioning accuracy ${ }^{[1][2]}$.

The establishment of fingerprint space determines the positioning range and positioning accuracy of Location Fingerprinting. Since the interpolation can reduce the number of sampling points, improve the efficiency of fingerprint space building and ensure the positioning accuracy, it becomes one of the research focuses on extending positioning range ${ }^{[3][4]}$. The major existing interpolation method to establish fingerprint space is the traditional Kriging ${ }^{[5]}$, log-propagation path loss model ${ }^{[6]}$ ${ }^{[7]}$ and the weighted distance inverse IDW ${ }^{[8]}$.The former two methods do not consider the relationship of sampling points, ignore the influences of the entire fingerprint spatial sampling points towards the measuring point correlation, resulting in lower interpolation accuracy. Traditional Kriging interpolation method needs Kriging interpolation calculation repeatedly to determine the Variogram model.

Therefore, proposing the Kriging interpolation algorithm that is relevant parameters theoretical model based on least squares fitting Variogram to create a fingerprint of space. Without increasing the number of sampling points, this method is to use the principle of least squares fitting Variogram model to calculate the parameters of the model. Experimental results show that the method by improving the fit of the experimental data to improve the positioning accuracy, better positioned for large regional environment.

\section{2 .Fingerprint spatial localization model}

Fig.1 shows the process of location fingerprinting, which is divided into two parts of the fingerprint space building and fingerprint comparing. The most basic task is to establish the fingerprint space. The intervals of sampling points that is grid spacing, determine the algorithm's accuracy. Establishing fingerprint space in a large area environment, the number of positioning fingerprints measurements require a substantial increase, which increase the workload, increasing grid spacing results in lower positioning accuracy. Therefore, the study focuses on the text 
interpolated reconstruction part, which can solve the problems of high complexity by larger positioning area and low positioning accuracy.

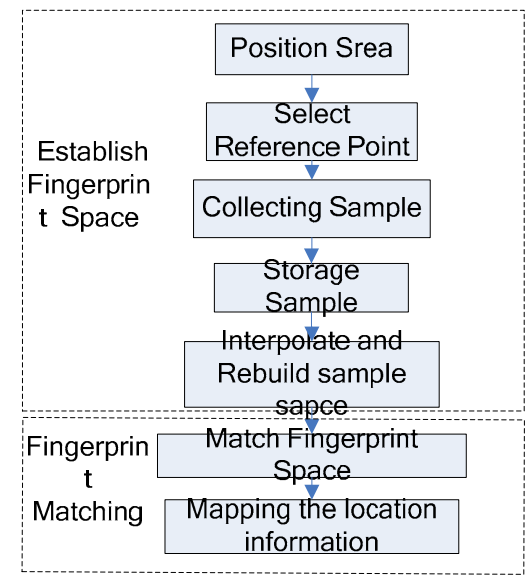

Figure 1.Fingerprint Positioning Process

In this paper, reconstruct the sample collection by improved Kriging interpolation algorithm. Improved Kriging interpolation method is improved Variogram that is the core of kriging interpolation method, using least-squares fit of the Variogram distribution curve calculated by actual measured value, to arrive higher fitting degree theory Variogram model expression.Thereby increasing the accuracy of Kriging interpolation method.

\section{3 .Kriging interpolation algorithm}

Kriging interpolation algorithm is spatial interpolation methods based on statistical Variogram model development. It is an unbiased, linear estimation method.

Variogram is proposed by describing spatial characteristics of the point to be measured. Its principle is to use the rules of research object changing with position to infer the characteristic value of the point to be measured. The experimental Variogram of the sample can be calculated by the equation is:

$$
\gamma^{*}(h)=\frac{1}{2 N_{h}} \sum_{i=1}^{N_{h}}\left[Z\left(x_{i}+h\right)-Z\left(x_{i}\right)\right]^{2}
$$

Where: $h$ is the separation distance between the two measurement points $N_{h}$ indicates the number of all points with the distance of $h$.

Kriging interpolation algorithm used the measured data of sample points and Equation(1) to calculate Variogram and draw the curve of Variogram. The model of Variogram used in the present fingerprint algorithm for spherical model

The equation is: $\gamma(h)=\left\{\begin{array}{l}C_{0}+C_{1}\left[1.5(h / a)-0.5(h / a)^{3}\right], 0 \leq h \leq a \\ C_{0}+C_{1}, h>a\end{array}\right.$

Where $C_{0}, C_{1}$, a are Undetermined coefficients.

Using Kriging method to interpolate in region is that, set $Z(x)$ for regional variables, and satisfy the second order stationary assumption, $Z\left(x_{i}\right)$ is the value of the spatial position point $x_{i}(i=1,2, \ldots n)$ in the region. The interpolate equation is:

$$
Z *\left(x_{0}\right)=\sum_{i=1}^{n} \lambda_{i} Z\left(x_{i}\right)
$$

Where: $Z^{*}\left(x_{0}\right)$ is the estimated value on the position point $x_{0}, Z\left(x_{i}\right)$ is the measurement value on the position point $x_{i}, \lambda_{i}$ is the weighting coefficient used to calculate $Z^{*}\left(x_{0}\right), \lambda_{i}$ selection criteria 
is to ensure an unbiased estimate and the estimated minimum variance. Make $E\left(Z^{*}-Z\right)=0$ to ensure unbiased estimate, from the starting intrinsic assumption condition, it was found $E(Z(x))$ is a constant giving relationship : $\sum_{i=1}^{n} \lambda_{i}=1$.In order to ensure a minimum estimate of variance, make $V_{\min }=\operatorname{Var}\left[Z^{*}\left(x_{0}\right)-Z\left(x_{0}\right)\right]$, and use the lagrange multiplication to calculate conditional extreme value. Get expression of Kriging equations containing $\mu, \lambda$ :

$$
\begin{aligned}
& \sum_{i=1}^{n} \gamma\left(x_{i}-x_{j}\right) \lambda_{i}+\mu=\gamma\left(x_{0}-x_{j}\right) \\
& \sum_{i=1}^{n} \lambda_{i}=1, j=1,2, \ldots, n
\end{aligned}
$$

Where : $\gamma\left(x_{i}-x_{j}\right)$ is the Variogram of $x_{i}$ and $x_{j}$. Solving equation to obtain the weight value $\lambda_{i}$, and bring it into equation 2 can obtain estimated value $Z *\left(x_{0}\right)$ that is the interpolation results.

\section{4 .Least-squares fit variogram}

For variogram curve fitting,this part giving the theoretical value of variogram parameters and theoretical models of variogram model. The specific method is to use the principle of least squares spherical fitting variogram model curve . By calculating get theoretical expressions of $C_{0}, C_{1}$, a, resulting get the theoretical expressions model.

Fitting spherical model is to fitting variogram under conditions of $0 \leq h \leq a$, that is: $\gamma(h)=C_{0}+C_{1}\left[1.5(h / a)-0.5(h / a)^{3}\right]$. where: $y=\gamma(h), \quad k_{0}=c_{0}, \quad k_{1}=\frac{3}{2} \frac{c_{1}}{a}, \quad k_{2}=\frac{1}{2} \frac{-c_{1}}{a^{3}}$, $x_{1}=h, x_{2}=h^{3}, \quad y^{*}=\gamma^{*}(h)$,so he above equation becomes:

$$
y=k_{0}+k_{1} x_{1}+k_{2} x_{2}
$$

So the fitting problem will be converted to binary linear regression of equation 4 . Determining factor of $k_{0}, k_{1}, k_{2}$ to determine the parameters $C_{0}, C_{1}$, a of model.Due to calculating the distance $h$ of variogram sample point is small, the greater the number of points for more accurate calculation, When the sample point distance increases the number of points is reduced, The actual value of the variogram of reduced reliability.So right value should change with the number of points varies so choose the right value $\omega=\mathrm{N}\left(\mathrm{h}_{\mathrm{i}}\right)$.According to the principle of weighted least squares get $\sum_{i=1}^{n}\left(N(i) y^{*}-N(i) y\right)^{2}=0$, then respectively partial derivative and obtained Solution of equations is:

$$
\left\{\begin{array}{l}
\mathrm{k}_{0}=\bar{y}-b_{1} \bar{x}_{1}-b_{2} \bar{x}_{2} \\
k_{1}=\left(L_{1 y} L_{22}-L_{2 y} L_{12}\right) /\left(L_{11} L_{22}-L_{12} L_{21}\right) \\
k_{2}=\left(L_{2 y} L_{11}-L_{1 y} L_{21}\right) /\left(L_{11} L_{22}-L_{12} L_{21}\right)
\end{array}\right.
$$

$$
\begin{gathered}
\text { Where: } \bar{y}=\sum_{i=1}^{n} N\left(h_{i}\right) y_{i} / \sum_{i=1}^{n} N\left(h_{i}\right), \bar{x}_{1}=\sum_{i=1}^{n} N\left(h_{i}\right) x_{1 i} / \sum_{i=1}^{n} N\left(h_{i}\right), \bar{x}_{2}=\sum_{i=1}^{n} N\left(h_{i}\right) x_{2 i} / \sum_{i=1}^{n} N\left(h_{i}\right) \\
L_{11}=\sum_{i=1}^{n} N\left(h_{i}\right)\left(x_{1 i}-\bar{x}_{1}\right)^{2}, L_{22}=\sum_{i=1}^{n} N\left(h_{i}\right)\left(x_{2 i}-\bar{x}_{2}\right)^{2}, L_{2 y}=\sum_{i=1}^{n} N\left(h_{i}\right)\left(x_{2 i}-\bar{x}_{2}\right)\left(y_{i}-\bar{y}\right) \\
L_{1 y}=\sum_{i=1}^{n} N\left(h_{i}\right)\left(x_{1 i}-\bar{x}_{1}\right)\left(y_{i}-\bar{y}\right), L_{2 y}=\sum_{i=1}^{n} N\left(h_{i}\right)\left(y_{i}-\bar{y}\right)^{2}, L_{12}=L_{21}=\sum_{i=1}^{n} N\left(h_{i}\right)\left(x_{1 i}-\bar{x}_{2}\right)\left(x_{2 i}-\bar{x}_{1}\right)
\end{gathered}
$$


then $c_{0}=k_{0}, c_{1}=\sqrt{-\frac{k_{1}}{3 k_{2}}}, a=\sqrt[3]{-\frac{4 k_{1}^{3}}{27 k_{2}}}$, and spherical model fitting variogram equation is :

$$
\gamma(h)=k_{0}+\sqrt{-\frac{k_{1}}{3 k_{2}}}\left[\frac{3}{2}\left(\frac{h}{\sqrt[3]{-\frac{4 k_{1}^{3}}{27 k_{2}}}}\right)+\left(\frac{27 h^{3} k_{2}}{8 k_{1}^{3}}\right)\right]
$$

(6)

In order to determine the degree level of different fitting methods to fit the experimental variograms . Chosen the goodness of fit to judge model. The equation is:

$$
R^{2}=\sum_{i=1}^{n}\left(\hat{y}_{i}-\bar{y}_{i}\right)^{2} / \sum_{i=1}^{n}\left(y_{i}-\bar{y}_{i}\right)^{2}
$$

where $: \hat{y}_{i}$ is the value of the use of spherical variogram model fitting, $\bar{y}_{i}$ is the average of the experimental variogram, $y_{i}$ is the value of experimental variogram.

\section{Implementation and Experimental Results}

The experimental site for open space, by field measurements obtained fingerprint information of the experimental area . Specific experimental scenario shown in Fig.2.

The topic chosen experimental launcher is signal generator,transmit power is $15 \mathrm{~dB}$, frequency is 800M.Transmitting antenna is Yagi antenna that the power lobe is 90 degrees, reception apparatus is spectrum analyzer, receiving antenna is a dipole antenna. This experiment is selected area of $100 *$ 100 within the scope.Specific methods is measuring the sampling points with way of grid measurement in area of 100 square meters, interval sampling points is $5 \mathrm{~m}$. Experiments collectes 400 sampling points. To ensure accurate measurements, each point measure five times,and take the average value as the final measurement data of each sampling point.

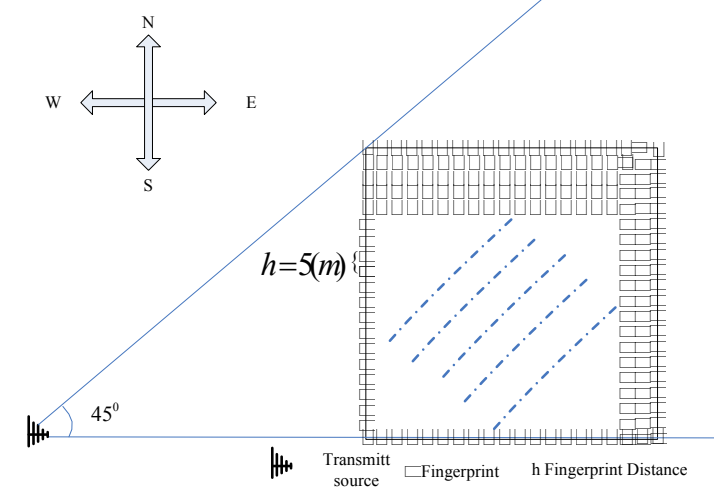

Figure2.Experimental Scene

\subsection{Experimental specific content}

Application of equation (8) and the obtained experimental variogram values that calculated from the spherical model parameters. To compare two method through the value of goodness of fit, and contrast the least squares fitting of Kriging interpolation with the traditional Kriging method, logarithmic propagation loss model interpolation and IDW,in part of signal strength interpolation error and the positioning error of fingerprint under the same conditions.

\section{2 .Experimental Analysis And Performance Comparison.}

According to the information of fingerprint and grid spacing of the fingerprint space, using equation (9) calculated the variogram values for each distance .

Through the experimental variogram values and the equation (10) and (11) to calculate and get the parameters $C_{0}=-0.21, C_{1}=2.35, a=79.63$ in east-west direction of the fitting Variogram spherical model and get the spherical model equation is: 


$$
\gamma(h)=-0.2+2.35\left[1.5(h / 79.63)-0.5(h / 79.63)^{3}\right]
$$

Using the traditional Kriging interpolation method obtained the spherical model expression is :

$$
\gamma(h)=-0.17+2.7\left[1.5(h / 85.3)-0.5(h / 85.3)^{3}\right]
$$

Through equation(12) get the $R=0.7961$ that is the goodness of fit of the spherical model calculated by improved Kriging. $R=0.6385$ is calculated by the traditional Kriging .The variogram model calculated by improved Kriging is closer to the distribution curve of experimental variogram.

The same with the east-west direction principle, the parameters of the spherical model in Southeast direction is $C_{0}=-0.27, C_{1}=5.07, a=97.87$. Take that parameters into the spherical model , and get expression is:

$$
\gamma(h)=-0.22+5.07\left[1.5(h / 97.87)-0.5(h / 97.87)^{3}\right]
$$

Using the traditional Kriging interpolation method obtained the spherical model expression is :

$$
\gamma(h)=-0.18+5.77\left[1.5(h / 105)-0.5(h / 105)^{3}\right]
$$

Through equation(13) get $R=0.8030$ that is goodness of fit of the spherical model calculated by improved Kriging. $R=0.7230$ is calculated by the traditional Kriging . By comparing the goodness of fit, in the southeast of the least squares methods fitting results closer to the actual curve.

In summary that shows the performance of improved Kriging is better than the traditional Kriging method.

Experimental comparison of interpolation error is that the test points were selected randomly in the test range and to compared the obtained error result between calculated the interpolation points and the actual value.Specific experiments is randomly selected 25 points that in 400 known measurement points for the point to be measured.

Fig.3 shows comparison of error between four kinds of interpolation methods and actual measurement,. When the grid spacing is 5 meters and 25 meters, improved Kriging compared with other methods the error is at least reduced by $0.17 \mathrm{~dB}-0.45 \mathrm{~dB}$. Experiments show that the perfromance of Kriging method base on least square fitting is better than other algorithms.

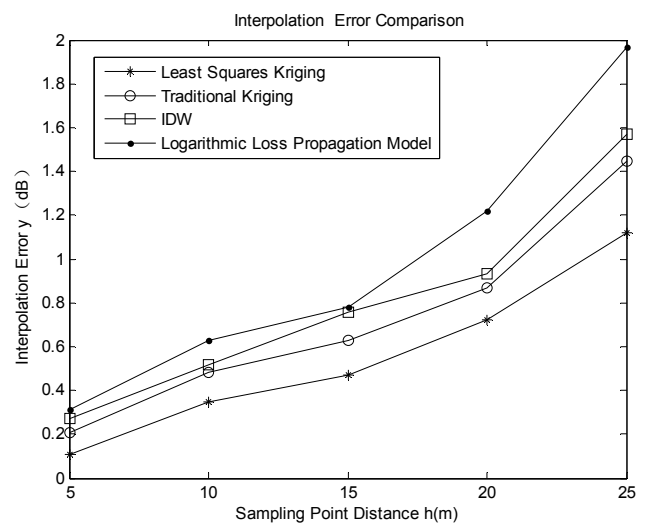

Figure 3.Interpolation Error Comparison

The contrast experiment of positioning error in location area of 100 square metres and selected 10 coordinates randomly for target positioning points.Using four interpolation methods to reconstruct fingerprint space.Specific reconfiguration method is the reconstructed 10 meters grid spacing of fingerprint space to 5 meters.And so on reconstructed the 30 meters grid spacing of fingerprint space to 15 meters.

The positioning process is that selected the Parameters $\mathrm{K}=3$ of $\mathrm{KNN}$ matching algorithm to position 10 target positinging points which have been selected in fingerprint space that be reconstructed of four kinds of interpolation methods .

Fig.4 shows the position error at four kinds of interpolation algorithm in different grid spacing.and In the same data conditions, the position error of fingerprint space be reconstructed by improved Kriging is significantly less than the other methods. The position error respectively is reduced by at least 0.4 meters and 1.47 meters in fingerprint space that grid spacing is reconstructed 
from 5 meters to 15 meters . Experiment shows that the fingerprint space is established by Kriging interpolation method based on the least squares fitting is better than the other three algorithms in the aspect of positioning.

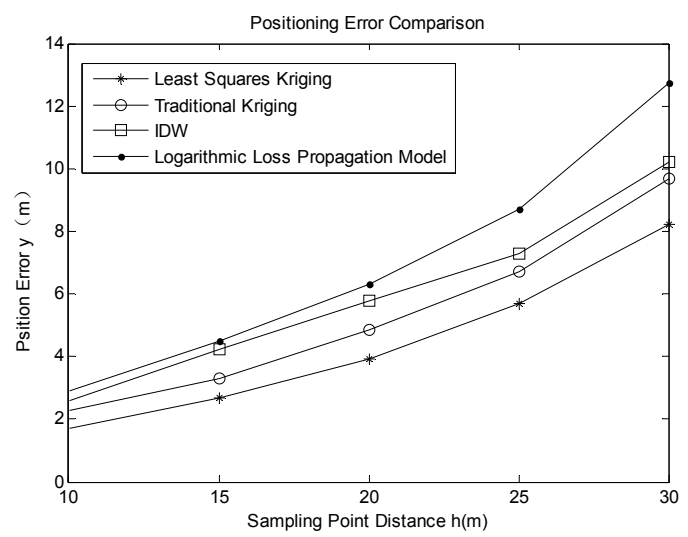

Figure 4.Position Error Comparison

\section{Conclusions}

The positioning accuracy of fingerprint localization algorithm is dependent on the fingerprint space deeply. The size of fingerprint space determines the scope of application of the location fingerprinting algorithm. This paper take the actual measurement data to analyzed different interpolation algorithms obtained the improved Kriging has great advantages. It is efficient in a wide area positioning environment to establish fingerprint space and can ensure the positioning accuracy, thus expand the Applicable scope of the location fingerprinting algorithm.

\section{References}

[1] Feng C, Au W S A, Valaee S, et al. Received-Signal-Strength-Based Indoor Positioning Using Compressive Sensing[J]. Mobile Computing, IEEE Transactions on, 2012, 11(12):1983 1993.

[2] Fang S, Lin T. A dynamic system approach for radio location fingerprinting in wireless local area networks[J]. Communications, IEEE Transactions on, 2010, 58(4):1020 - 1025.

[3] Chen Y, Yang Q, Yin J, et al. Power-efficient access-point selection for indoor location estimation[J]. Knowledge and Data Engineering, IEEE Transactions on, 2006, 18(7):877 - 888.

[4] Liu X, Zhang S, Lu H, et al. Method for efficiently constructing and updating radio map of fingerprint positioning[C]. GLOBECOM Workshops (GC Wkshps), 2010 IEEE. IEEE, 2010:74 - 78.

[5] Li B, Wang Y, Lee H K, et al. Method for yielding a database of location fingerprints in WLAN[J]. Communications, IEE Proceedings-, 2005, 152(5):580 - 586.

[6] Yin J, Yang Q, Ni L M. Learning Adaptive Temporal Radio Maps for Signal-Strength-Based Location Estimation[J]. Mobile Computing, IEEE Transactions on, 2008, 7(7):869 - 883.

[7] Yang J, Chen Y. Indoor Localization Using Improved RSS-Based Lateration Methods[C]. Global Telecommunications Conference, 2009. GLOBECOM 2009. IEEE. IEEE, 2009:1 - 6.

[8] Kuo S, Tseng Y. Discriminant Minimization Search for Large-Scale RF-Based Localization Systems[J]. Mobile Computing, IEEE Transactions on, 2011, 10(2):291 - 304. 